\title{
Flammer Syndrome, A Potential Risk Factor for Central Serous Chorioretinopathy?
}

\author{
Tatjana Josifova ${ }^{1 *}$, Franz Fankhauser ${ }^{1}$ and Katarzyna Konieczka ${ }^{1,2}$ \\ ${ }^{1}$ Augenzentrum Prof Fankhauser, Bern, Switzerland \\ ${ }^{2}$ Universitätspital Basel, Augenklinik, Basel, Switzerland \\ *Corresponding author: Tatjana Josifova, Augenzentrum Prof Fankhauser, Bern, Switzerland
}

\section{ARTICLE INFO}

Received: 幽 December 16, 2019

Published: 㓞January 06, 2020

Citation: Tatjana Josifova, Franz Fankhauser, Katarzyna Konieczka. Flammer Syndrome, A Potential Risk Factor for Central Serous Chorioretinopathy?. Biomed J Sci \& Tech Res 24(2)-2020. BJSTR. MS.ID.004026.

Keywords: Central Serous Chorioretinopathy; Flammer Syndrome; Primary Vascular Dysregulation; Retinal Venous Pressure

\begin{abstract}
Central serous chorioretinopathy (CSCR) is forth most common retinal disease, mostly affecting men in their third and fourth life decade. Changes most often involve the macula and are associated with pigment epithelial and neurosensory retinal detachment. The literature highlights an involvement of the choroidal veins and pigment epithelium in the pathogenesis of CSCR. Nevertheless, both the risk factors and the molecular mechanisms of CSCR remain uncertain. The Flammer syndrome refers to a phenotype characterized by the combination of primary vascular dysregulation with a cluster of additional symptoms and signs. Subjects affected by the syndrome have a predisposition to react differently to a number of stimuli, such as cold, physical or emotional stress, or high altitude. We postulate that Flammer syndrome might be one of the risk factors for CSCR. This relationship, however, requires further investigation. Better knowledge of risk factors, such as Flammer syndrome, will potentially help to prevent the disease in the future.
\end{abstract}

Abbreviations: BRB: Blood-Retinal Barrier; CSCR: Central Serous Chorioretinopathy; RPE: Retinal Pigment Epithelial; PE: Pigment Epithelial; VA: Visual Acuity; DRPE: Diffuse Retinal Pigment Epitheliopathy; CNV: Choroidal Neovascularization; FA: Fluorescein Angiography; ICG: Indocyanine Green; OCT: Optical Coherence Tomography; FS: Flammer Syndrome; RVP: Retinal Venous Pressure

\section{Introduction}

The blood-retinal barrier (BRB) is composed of an inner and outer barrier. Its structure controls the diffusion of molecules along different pathways. The role of the BRB is to prevent the entry of large molecules of the circulating blood into the retina and into the tight junctions between retinal epithelial cells. It also prevents the passage of large molecules from the fenestrated choriocapillaris into the retina. The function of the BRB is regulated by molecules such as prostaglandins, nitric oxide, or endothelin. Endothelin is a strong vasoconstrictor which also dysregulates the BRB function. Regulation and dysregulation of barrier functions, as well as, regulation and dysregulation of the size of vessels, can lead to different pathological conditions in the eye. One of these is CSCR. When the weakening of the BRB on the level of the pigment epithelium allows the diffusion of molecules into the retina, the result is a clinical picture of a CSCR. In this review, we discuss a potential relationship between primary vascular dysregulation [1], the core sign of FS [2,3], and barrier dysregulation, the core sign of CSCR. This is based on both published literature and clinical observation of our own patients.

\section{Central Serous Chorioretinopathy}

Central serous chorioretinopathy (CSCR) is the fourth most common retinopathy after age-related macular degeneration, diabetic retinopathy, and retinal vein occlusions. Many epidemiology studies have demonstrated a higher incidence of the disease among the male population in their third and fourth decades [4]. Numerous risk factors for CSCR have been reported, although the cause of the disease remains unknown. One of the best-known risk factors is the use of glucocorticoids. This theory supports the increased 
incidence of CSCR in patients with Cushing's syndrome and those who are pregnant [5]. In both conditions, the endogenous plasma cortisol levels are elevated, which can influence the choroidal circulation. Among the risk factors for CSCR, one of the most frequently discussed is psychological stress. The Type A personality has frequently been found to be associated with CSCR. Yannuzzi's study of CSCR and personality types supports this association [6].

Additional factors, such as the use of psychotropic and sympathomimetic medication; systemic factors such as gastroesophageal reflux and autoimmune disorders; the use of tobacco and alcohol are also thought to be potential risks in the pathogenesis of CSCR [7-9]. To study the choroidal circulation of CSCR patients, C. Prünte und J. Flammer in 1996 performed indocyanine-green angiography with a scanning laser ophthalmoscope and digital imaging analysis. They found in these patients a delayed arterial filling followed by capillary and venous congestion. This observation supports our hypothesis of an involvement of primary vascular dysregulation in the pathogenesis of this disease [10].

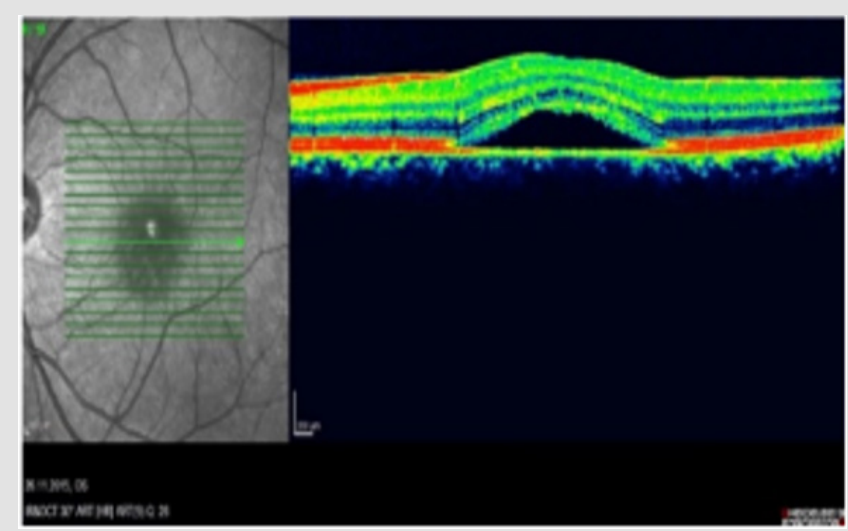

Figure 1: Autofluorescence and OCT findings of fluid accumulation in acute CSCR form.

Phenomenology of CSCR: CSCR is a chronic, recidivist disorder affecting the macula and it could be characterized by serous neurosensory retinal detachment and/or retinal pigment epithelial (RPE) detachment. One of the most common disease signs is serous pigment epithelial detachment (PED); this can occur as a single event or as multiple small PEDs. Pigment epithelial (PE) changes can also exist without the splitting or thickening of the RPE complex or changes in the neurosensory retina. On the other hand, the accumulation of fluid in the subretinal space is produced by an abnormal focal defect at the level of the RPE [11]. CSCR can occur in an acute or a chronic form. Recent clinical trials have considered CSCR to be in a chronic form when there has been persistent fluid accumulation for at least three months. In the primary appearance of the disease, most RPE and neurosensory changes resolve spontaneously within 2-3 months (Figures 1 \& 2). Recidivism is common and can be accomplished by persistent RPE changes and decrease in visual acuity (VA). Most patients describe the symptoms as the appearance of a dark area in the center of the vision. As the disease progresses, straight lines may appear bent, crooked or irregular [12]. The dark areas are present in every recidivism, and in the chronic phase can lead to central blind spots. Prognosis is highly dependent on the presenting VA; patients with initial good VA have a better prognosis compared to patients with poor initial VA. Every recidivism is associated with further VA loss. Recovery time for average two to three Snellen lines can last for several years [13].

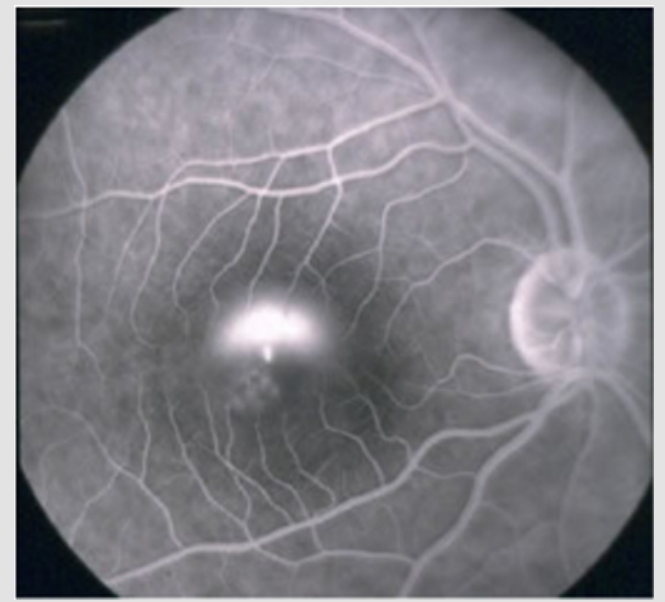

Figure 2: Fluorescein Angiography: "Smoke like"hyper fluorescence in acute CSCR form.

Widespread RPE damage in CSCR can provoke diffuse retinal pigment epitheliopathy (DRPE) [14]. Over time, this can lead to photoreceptor death which, together with PE death, may result in permanent visual loss. Ischemic changes in severe cases could be followed by choroidal neovascularization (CNV) and irreversible visual loss due to permanent fluid accumulation and cell death. Choroidopathy with or without choroidal infarction can stimulate ischemic ocular changes and consecutive CNV. The systemic or local factors that influence the course of the disease, its ischemic changes, or the poor prognosis of some cases remain unknown. [15]. The status of the retinal pigment epithelium distinguishes the first, or classic, form of CSCR from the second form, which is marked by diffuse retinal pigment epitheliopathy (DRPE) [16]. Compared to the classic form, characterized by minimal changes at the level of the RPE, DRPE cases have extensive RPE damage and long lasting subretinal fluid accumulation The third form, a bullous, very rare variant of CSCR is associated with poor prognosis because of inferiorly gravitating fluid and progressive RPE damage. That this form occurs largely beneath the fovea is another reason it is associated with visual loss [17]. The use of current diagnostic tools such as fluorescein angiography (FA) and indocyanine green (ICG), as well as novel developments including optical coherence tomography (OCT), contribute to a deeper understanding and follow-up of the disease.

Pathogenesis of CSCR: The role of choroid has been emphasized as one of the most important factors in the pathogenesis 
of CSCR. Choroidal blood-flow changes can be induced through the production of free radicals, inflammatory cells, and nitric oxide. Long-standing choroidal changes and vein congestion together with the extensive dysfunction and loss of RPE, unable the resorption of the subretinal fluid [18]. Hyperpermeable choroidal vessels produce increased tissue hydrostatic pressure, and lead to the breakdown of the blood-retinal barrier. This promotes PEDs, subretinal fluid accumulation, or neurosensory detachment. The reduced reflectivity between the outer choroid and sclera, evident in the OCT findings, are a product of choroidal venous congestion. The choroidal vascular dysregulation detected in the OCT are also seen in the delay of choroidal filling apparent in the FA. This delay could be caused by choroidal lobular ischemia [19]. The ICG findings show early filling and dye wash out, accomplished with late persistent hyper fluorescence. Using the laser interferometer, Tittle and colleagues compared the fundus pulsation amplitudes in CSCR eyes and control eyes. In the affected eyes of CSCR patients, they measured greater amplitudes compared to the non-affected eyes of the same patients. Compared to the control subjects with similar systemic parameters, a significant difference was noticed in the laser interferometer results of the CSCR patients. Nevertheless, in the inactive phase of the disease, the patients with CSCR still had increased sub foveal blood flow in the affected eye compared to the non-affected eye. The same results were found when compared to the control subjects [20]. Spectral domain OCT (SD-OCT) shows that, because of choroidal-flow dysregulation, the thickened choroid evinces a perfusion delay and choroidal ischemia, which is a target factor for the consecutive retinal changes [21,22]. It is uncertain whether choroidopathy and epitheliopathy have a common pathogenetic mechanism that can indicate the progression and prognosis of the disease. For example, not every patient with DRPE and permanent visual loss because of epitheliopathy, has a choroidopathy in terms of subretinal fluid presence, on the OCT findings [23].

\section{Flammer Syndrome}

The term Flammer syndrome (FS) [1-3] describes a phenotype characterized by the presence of primary vascular dysregulation together with a cluster of additional symptoms and signs that may occur in healthy people as well as in people with disease. It refers to a predisposition to react differently to a number of stimuli, such as cold [24], physical or emotional stress, or high altitude. The most common symptom or sign is cold extremities with an increased response to coldness [24]. These subjects often have cold hands and/or feet, both subjectively and objectively. The extremities can be cold even in normal environmental temperatures but are particularly likely to respond with vasoconstriction to coldness or psychological or mechanical stress. These subjects often have prolonged sleep onset time [24], as temperature and sleep are interrelated. One can fall asleep only after the extremities are warmed up, and this often takes longer in FS subjects. The second most common sign of FS is low blood pressure [25], especially when the subjects are young.

Blood pressure can be generally low or can drop when subjects stand up (orthostatic hypotension) or when they are sleeping (nocturnal over-dipping). FS subjects have a reduced feeling of thirst. They, however, normally drink enough, as they know they need to do so. These subjects are characterized by an enhanced sensitivity to many types of stimuli. They have increased sensitivity to certain drugs (e.g., calcium channel blockers and systemic betablockers). They also have increased pain sensation, increased sense of smell [26], increased sensitivity to high altitude, etc. They often suffer from tinnitus and sometimes even from sudden (mostly reversible) hearing loss [27]. The syndrome occurs more often in females than males, in subjects who are slim more than in those who are obese [28], in academics more than in blue-collar workers, and in Asians more than in Caucasians.

Typically, these persons have a tendency toward perfectionism. In terms of ocular perfusion, FS subjects have reduced autoregulation [29]. This explains why in these cases ocular blood flow correlates with finger blood flow. These subjects have increased retinal venous pressure, stiffer retinal vessels [30], reduced neurovascular coupling [31], and optic disc hemorrhages [32]. They also have slightly increased ET-1 plasma levels and altered gene expression in circulating lymphocytes. FS is related to some eye diseases, such as glaucoma, especially normal tension glaucoma [33,34], retinitis pigmentosa [35-37], Leber's hereditary optic neuropathy [1], and optic nerve compartment syndrome [1,38]. FS is also a risk factor for vascular occlusions; those that have been reported include retinal vein occlusions [39,40], cilioretinal artery occlusions [41], choroidal infarctions [42] anterior ischemic optic neuropathies, and Susac syndrome [43]. FS is also more often present in Multiple Sclerosis patients [44] and is a risk factor for perioperative visual loss $[45,46]$, breast cancer and metastatic disease $[47,48]$.

\section{Flammer Syndrome: A Potential Risk Factor for CSCR}

While the cause of CSCR remains unclear, a number of risk factors have been proposed, such as a Type A personality. Emotional stress seems to be an important parameter and a risk factor for a CSCR development. Individuals with FS typically respond to stress similarly, with transient vasoconstriction and/or vasodilatations. Indeed, our CSCR patients often indicated cold hands or feet under stress. In addition, they had other symptoms of FS, such as a reduced feeling of thirst and prolonged sleep onset time or low blood pressure.

The core of FS is a primary vascular dysregulation. A dysregulation of choroidal veins in CSCR was first observed by Prünte and then confirmed by several studies $[10,49]$. Interestingly, most of our CSCR patients also had an increased retinal venous pressure (RVP) (contact lens dynamometer [4], IMEDOS Systems UG, Jena, Germany), as it has also been described in FS subjects. An 
elevated RVP is most often a result of a local venous constriction at the level of the optic nerve head. This, in turn leads to a dilatation of the retinal veins upstream [50]. A similar mechanism could occur in the choroidal veins of CSCR patients.

We also observed increased retinal venous pressure in the nonaffected fellow eyes of our CSCR patients. The same behavior has been described among patients with retinal vein occlusion, who also experienced an increase in retinal venous pressure in the clinically non-affected fellow eye [51]. In CSCR patients, the thickened choroid can be explained by the focal or diffuse dilatation of large choroidal vessels. ICG angiography has shown that these dilated vessels are commonly localized within areas of increased choroidal vascular permeability [52]. Above these dilated choroidal vessels, the inner choroidal layer, which includes medium and small vessels, is thinner in the area of a barrier dysfunction of the RPE, than in adjacent areas. Furthermore, we observed a transient increase of plasma endothelin-1 during the acute phase of CSCR. The local concentration of endothelin-1 may even be considerably higher in these cases and could explain both the dysregulation of choroidal vessels and the disruption of the RPE barrier [53]. A transient increase of plasma endothelin-1 level has also been observed in FS subjects after provocation by cold. The vascular dysregulation also leads to an unstable oxygen supply and thereby increases oxidative stress, further damaging the barrier function. Although FS occurs more often in young females, CSCR is more frequent in young males. We assume that for CSCR a certain level of testosterone might be a "condition sine qua non" [54].

\section{Conclusion}

CSCR is a recidivist, sight-threatening disease of origins yet unknown. Therapeutic options are limited. Because a relationship appears to exist between the disease and corticosteroids, the discontinuation of steroid treatment is advised among those with CSCR. That reducing stress is beneficial in its treatment is generally agreed upon. If FS is confirmed to be a risk factor, treatment of FS may help to prevent CSCR and/or mitigate the course of the disease.

\section{References}

1. Flammer J, Konieczka K, Flammer AJ (2013) The primary vascular dysregulation syndrome: implications for eye diseases. EPMA J 4(1): 14.

2. Konieczka K, Robert Ritch, Carlo Enrico Traverso, Dong Myung Kim, Michael Scott Kook, et al. (2014) Flammer syndrome. EPMA J 5(1): 11.

3. Flammer J, Konieczka K, Bruno RM, Virdis A, Flammer AJ, et al. (2013) The eye and the heart. Eur Heart J 34(17): 1270-1278.

4. Kitzmann AS, Pulido JS, Diehl NN, Hodge DO, Burke JP, et al. (2008) The incidence of central serous chorioretinopathy in Olmsted County, Minnesota, 1980-2002.0phthalmology 115(1): 169-173.

5. Bouzas EA, Scott MH, Mastorakos G, Chrousos GP, Kaiser Kupfer MI, et al. (1993) Central serous chorioretinopathy in endogenous hypercortisolism. Arch Ophthalmol 111(9): 1229-1233.

6. Yannuzzi LA (1987) Type A behavior and central serous chorioretinopathy. Retina 7(2): 111-131.
7. Tittl MK, Spaide RF, Wong D, Pilotto E, Yannuzzi LA, et al. (1999) Systemic findings associated with central serous chorioretinopathy. Am J Ophthalmol 128(1): 63-68.

8. Mansuetta CC, Mason JO, Swanner J, Feist RM, White MF Jr, et al. (2004) An association between central serous chorioretinopathy and gastroesophageal reflux disease. Am J Ophthalmol 137(6): 1096-1100.

9. Spaide RF, Campeas L, Haas A, Yannuzzi LA, Fisher YL, et al. (1996) Central serous chorioretinopathy in younger and older adults. Ophthalmology 103(12): 2070-2079.

10. Prünte C, Flammer J (1996) Choroidal capillary and venous congestion in central serous chorioretinopathy. Am J Ophthalmol 121(1): 26-34.

11. Jirarattanasopa $P$, Ooto $S$, Tsujikawa A, Yamashiro $K$, Hangai $M$, et al. (2012) Assessment of macular choroidal thickness by optical coherence tomography and angiographic changes in central serous chorioretinopathy. Ophthalmology 119(8): 1666-1678.

12. Ross A, Ross AH, Mohamed Q (2011) Review and update on central serous chorioretinopathy. Curr Opin Ophthalmol 22(3): 166-173.

13. Yoshioka H (1991) The etiology of central serous chorioretinopathy. Arch Ophthalmol 32: 128-129.

14. Wang M, Sander B, la Cour M, Larsen M (2005) Clinical characteristics of subretinal deposits in central serous chorioretinopathy. Acta Ophthalmol Scand 83(6): 691-696.

15. Yannuzzi LA (2010) Central serous chorioretinopathy: A personal perspective. Am J Ophthalmol 149(3): 361-363.

16. Spaide RF, Campeas L, Haas A (1996) Central serous chorioretinopathy in younger and older adults. Ophthalmology 103(12): 2070-2079.

17. Gass JD (1973) Bullous retinal detachment: An unusual manifestation of idiopathic central serous choroidopathy. Am J Ophthalmol 75(5): 810821.

18. Iida T, Kishi S, Hagimura N, Shimiizu K (1999) Persistent and bilateral choroidal vascular abnormalities in central serous chorioretinopathy. Retina 19(6): 508-512.

19. Okushiba U, Takeda M (1997) Study of choroidal vascular lesions in central serous chorioretinopathy using indocyanine green angiography. Nippon Ganka Gakkai Zasshi 101(1): 74-82.

20. Tittl M, Polska E, Kircher K, Andreas Kruger, Noemi Maar, et al. (2003) Topical fundus pulsation measurement in patients with active central serous chorioretinopathy. Arch Ophthalmol 121: 975-978.

21. Yang L, Jost J, Wenbin W (2013) Choroidal vessel diameter in central serous chorioretinopathy. Acta Ophthalmol 91(5): e358-e362.

22. Van Ventholven MEJ, Garcia PM, Rosen RB, De Smet (2005) Evaluation of the central serous chorioretinopathy with a face optical coherence tomography. Br J Ophthalmol 89(11): 1483-1488.

23. Maruko I, Iida T, Sugano Y, Ojima A, Sekiryu T Subfoveal, et al. (2011) Choroidal thickness in fellow eyes of patients with central serous chorioretinopathy. Retina 31(8): 1603-1618.

24. Saner H, Wurbel H, Mahler F, J Flammer, P Gasser, et al. (1987) Microvasculature evaluation of vasospastic syndromes. Adv Exp Med Biol 220: 215-218.

25. Gherghel D, Orgul S, Gugleta K (2001) Retrobulbar blood flow in glaucoma patients with nocturnal over-dipping in systemic blood pressure. Am J Ophthalmol 132(5): 641-647.

26. Mozaffarieh M, Hauenstein D, Schoetzau A, Katarzyna Konieczka, Josef Flammer, et al. (2010) Smell perception in normal tension glaucoma patients. Mol Vis 16: 506-510.

27. Palmer KT, Griffin MJ, Syddall HE, B Pannett, C Cooper, et al. (2002) Raynaud's phenomenon vibration induced white finger and difficulties in hearing. Occup Environ Med 59(9): 640-642. 
28. Mozaffarieh M, Fontana Gasio P, Schotzau A, Selim Orgül, Josef Flammer, et al. (2010) Thermal discomfort with cold extremities in relation to age gender and body mass index in a random sample of a Swiss urban population. Popul Health Metr 8: 17.

29. Gherghel D, Orgul S, Dubler B, Patricia Lübeck, Konstantin Gugleta, et al. (1999) Is vascular regulation in the central retinal artery altered in persons with vasospasm? Arch Ophthalmol 117: 1359-1362.

30. Oettli A, Gugleta K, Kochkorov A, Katamay R, Flammer J, et al. (2011) Rigidity of retinal vessel in untreated eyes of normal tension primary open-angle glaucoma patients. J Glaucoma 20(5): 303-306.

31. Gugleta K, Zawinka C, Rickenbacher I, Kochkorov A, Katamay R, et al. (2006) Analysis of retinal vasodilation after flicker light stimulation in relation to vasospastic propensity. Invest Ophthalmol Vis Sci 47(9): 4034-4041.

32. Grieshaber MC, Terhorst T, Flammer J (2006) The pathogenesis of optic disc splinter haemorrhages: A new hypothesis. Acta Ophthalmol Scand 84(1): 62-68.

33. Flammer J, Orgül S, Costa VP, Orzalesi N, Krieglstein GK, et al. (2002) The impact of ocular blood flow in glaucoma. Prog Retin Eye Res 21(4):359393.

34. Koniezcka K, Hyuk Jin Choi, Simone Koch, Franz Fankhauser, Andreas Schoetzau, et al. (2017) Relationship between normal tension glaucoma and Flammer syndrome. EPMA J 8(2): 111-117.

35. Konieczka K, Flammer AJ, Todorova M, Meyer P, Flammer J, et al. (2012) Retinitis pigmentosa and ocular blood flow. EPMA J 3(1): 17-19.

36. Konieczka K, Koch S, Schoetzau A, Todorova MG (2016) Increased prevalence of Flammer syndrome in patients with retinitis pigmentosa. Klin Monbl Augenheilkd 233(4): 448-452.

37. Todorova MG, Josifova T, Konieczka K (2016) Endothelin-1 plasma levels in patients with both retinitis pigmentosa and Flammer syndrome. Klin Monbl Augenheilkd 232: 514-518.

38. Konieczka K, Todorova MG, Bojinova R, Binggeli T, Chackanthayil TN, et al. (2016) Unexpected effect of calcium channel blockers on the optic nerve compartment syndrome. Klin Monbl Augenheilkd 233(4): 387390.

39. Frenkl SA, Mozaffarieh M, Flammer J (2010) Retinal vein occlusions: The potential impact of a dysregulation of the retinal veins. EPMA J 1(2): 253-261.

40. Flammer J, Konieczka K (2015) Retinal venous pressure: The role of endothelin. EPMA J 6: 21

41. Konieczka, K, Todorova MG, Chackathayil TN, Henrich PB (2015) Cilioretinal artery occlusion in a young patient with Flammer syndrome and increased retinal venous pressure. Klin Monbl Augenheilkd 232(4): 576-578.

42. Terelak Borys B, Grabska Liberek I, Piekarniak Wozniak A, Konieczka $\mathrm{K}$ (2017) Choroidal infarction in a glaucoma patient with Flammer syndrome. BMC Ophthalmol 17: 23.

43. Flammer J, Kaiser H, Haufschild T (2001) Susac syndrome: A vasospastic disorder? Eur J Ophthalmol 11(2): 175-179.

44. Koniezcka K, Koch S, Binggeli T, Schoetzau A, Kesselring J, et al. (2016) Multiple sclerosis and primary vascular dysregulation (Flammer syndrome). EPMA J 7: 13.

45. Bojinova RI, Konieczka K, Todorova MG (2016) Unilateral loss of vision after spinal surgery in a patient with Flammer syndrome. Klin Monbl Augenheilkd 233(4): 429-431.

46. Bojinova RI, Konieczka K, Meyer P, Todorova MG (2016) The trilateral link between anesthesia perioperative visual loss and Flammer syndrome. BMC Anesthesiol 16: 10.

47. Zubor P, Gondova A, Polivka J, Kasajova P, Konieczka K, et al. (2017) Breast cancer and Flammer syndrome: any symptoms in common for prediction, prevention and personalised medical approach? EPMA J 8(2): 129-140.

48. Bubnov R, Polivka J, Zubor P, Konieczka K, Golubnitschaja, et al. (2017) O. "Pre-metastatic niches" in breast cancer: are they created by or prior to the tumour onset? "Flammer Syndrome" relevance to address the question. EPMA J 8(2): 141-157.

49. Nicholson B, Noble J, Forooghian F, Meyerle C (2013) Central serous chorioretinopathy: Update on pathophysiology and treatment. Surv Ophthalmol 58(2): 103-126.

50. Flammer J, Koniezcka K (2015) Retinal venous pressure: the role of endothelin. EPMA J 6: 21.

51. Mozaffarieh M, Bätschi M, Henrich PB, Schoetzau A, Flammer J, et al. (2014) Retinal venous pressure in the non-affected eye of patients with retinal vein occlusions. Graefes Arch Clin Exp Ophthalmol 252(10): 1569-1571.

52. Jirarattanasopa $\mathrm{P}$, Ooto $\mathrm{S}$, Tsujikawa A, Yamashiro $\mathrm{K}$, Hangai $\mathrm{M}$, et al. (2012) Assessment of macular choroidal thickness by optical coherence tomography and angiographic changes in central serous chorioretinopathy. Ophthalmology 119(8): 1666-1678.

53. Salvatore S, Vingolo EM (2010) Endothelin-1 role in human eye: A review. J Ophthalmol p. 10.

54. Grieshaber MC, Staub JJ, Flammer J (2007) The potential role of testosterone in central serous chorioretinopathy. Br J Ophthalmol 91(1): 118-119.

\section{ISSN: 2574-1241}

DOI: $10.26717 /$ BJSTR.2020.24.004026

Tatjana Josifova. Biomed J Sci \& Tech Res

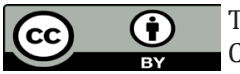

This work is licensed under Creative Commons Attribution 4.0 License

Submission Link: https://biomedres.us/submit-manuscript.php

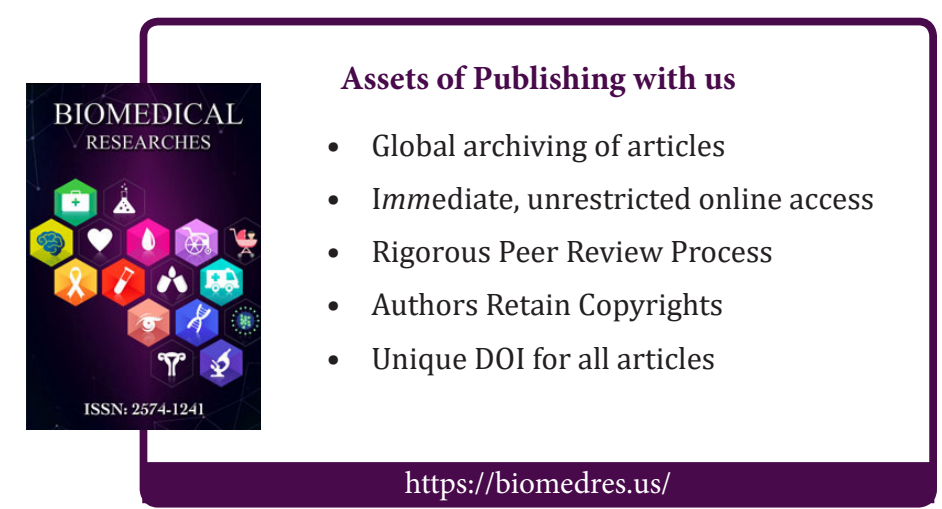

